\title{
ERROR ESTIMATION AND ADAPTIVITY
}

\author{
Pierre J. Beckers \& Hai G. Zhong \\ LTAS-Infographie, University of Liege \\ 21 rue Ernest-Solvay, 4000 Liège, Belgium
}

\begin{abstract}
A review of stress computation methods is presented for finite element displacement models. They include an original stress smoothing method, called "averaging + extrapolation", based on the idea of "optimal stress extrapolation points"; it is valid for general two dimensional meshes composed of iso-parametric elements of degree up to three. The smoothed stress field is used to derive an a posteriori error estimator measured in the energy norm. Numerical examples in plane elasticity are presented to show the reliability of the error estimator and to introduce its use in adaptive meshing.
\end{abstract}

\section{STRESS COMPUTATION FOR FINITE ELEMENT DISPLACEMENT MODELS}

For displacement models, stress computation is the most important post-processing operations. The methods of stress computation can be classified into three main types according to their order of complexity [1]:

1. direct computation;

2. direct computation followed by smoothing, including averaging, extrapolation, fitting, projection, iteration and other post-processing operations;

3. indirect computation by using specially constructed test functions, called extraction functions.

Methods of type 1) and 2) are simple and straightforward. They are available in most finite element softweres. Methods of type 3) are recent and not yet generally available. While the latter methods require somewhat more work in the post-solution phase than methods of type 1) and 2), the additional work is more than compensated for by the high efficiency of these methods, allowing the use of mostly fewer degrees of freedom to get required levels of accuracy.

In the following paragraphs, the direct computation procedure will be defined. A review of the methods of type 2) will be given. For more details on the methods of type 3), see [2].

\subsection{Direct computation}

The finite element solution $u_{k}$ of a displacement model is represented by the following relation:

$$
u_{k}=N q
$$

where $u_{h}$ is the displacement field, $N$ the matrix of shape functions and $q$ the nodal displacement vector. The finite element strain field is easily derived:

$$
\varepsilon_{n}=L u_{k}=B q
$$

where $L$ is a differential operator, $B=L N$ is the strain-nodal displacement matrix. The direct finite element stress field is computed by using the following formula

$$
\sigma_{k}=H \varepsilon_{k}=T g
$$

where $H$ is the stress-strain matrix. $T=H B$ is the stress matrix related to nodal displacements. It is available 
anywhere but generally calculated at the Gauss points of each element.

The global nodal displacement vector $q$ is obtained by solving the following system of equations

$$
K \boldsymbol{q}=\boldsymbol{g}
$$

where $K$ is the global stiffness matrix, $g$ is the global nodal force vector.

The finite element stress field $\sigma_{h}$ directly computed from (3) is generally discontinuous across element interfaces. It does not satisfy the equilibrium conditions neither in the interior nor on the boundaries of the structure. It is reliable only at the global level, in a certain mean sense.

\subsection{General form of smoothed stress fields}

Stress smoothing has long been a source of difficulty in the analyses based on displacement formulation. A review of stress smoothing methods used in the early finite element applications can be found in [3]. Even now the problem has not yet been solved with full satisfaction. Some stress smoothing methods, which are or have been largely used in the practice, will be presented below.

In many circumstances, such as in graphical representation of finite element stress fields and in estimation of discretization error, it is interesting to obtain a continuous stress field that is expected to be more precise than the direct one. This can be achieved in two steps.

First, the key point of the method is to estimate the stresses at certain points $j\left(j=1, \ldots, m_{n o d}\right)$. They are generally chosen as nodes corresponding to a degree $q$ which may be different from $p$, degree of the shape functions of the finite element model. This allows to define $s:$ the vector of estimated nodal stresses.

Secondly, by using a standard finite element interpolation, estimated stresses at any point can be evaluated by the following formula

$$
\tilde{\sigma}=M s
$$

where $\boldsymbol{M}$ is called stress smoothing matrix, which contains standard shape functions.

\subsection{Estimated nodal stresses}

\subsubsection{Simple elemental averaging}

For most commercial finite element programs, stresses are only evaluated at the Gauss points used in the numerical integration of elemental stiffness matrices. In general, these programs output also the element mean stresses derived from the Gauss points. Such element mean stresses are superconvergent in a mean sense.

An easy and cheap way of obtaining a smoothed stress field is to calculate the mean stresses at a node from the mean stresses of the surrounding eiements. Because of its simplicity and low cost, the method of simple elemental averaging is still used in most commercial finite element programs. The smoothed stresses at boundary nodes are generally highly under- or over-estimated. However, it is generally in these portions of structures that greater attention has to be paid by engineers.

\subsubsection{Simple nodal averaging}

Another stress smoothing method, which is a little more expensive than the preceding one, consists in averaging element nodal stresses from the stress tensor evaluated at node $j$ of element $e$. Stresses at such points are generally not calculated in the commercial programs but can be routinely obtained in the post-processing phase. The resulting smoothed stress field is generally more precise than the one obtained by the method of simple elemental averaging.

This method, called the simple nodal averaging method, is still less precise for boundary nodes. For non-uniform meshes it is not very reliable because it does not consider the size of the surrounding elements. 
This method is a special case of the more general projection method characterized by the following property:

$$
\int_{h}\left(\widetilde{\sigma}-\sigma_{\star}\right)^{T} P d \Omega=0
$$

where $P$ is a stress projection matrix, and $\Omega$ the domain of the structure. For the method of simple nodal averaging, the projection matrix is composed of nodal Dirac functions.

\subsubsection{Inverse finite element method}

The finite element method is a procedure of minimizing the global stress error measured in a weighted $L_{2}$-norm. The stress field $\sigma_{A}$ is obtained by the following minimization procedure

$$
\text { Minimize }\left|\sigma-\sigma_{k}\right|_{H}^{2}=\int_{h}\left(\sigma-\sigma_{k}\right)^{T} H^{-1}\left(\sigma-\sigma_{h}\right) d \Omega
$$

where $\sigma$ is the exact stress field, $\left.I \cdot\right|_{H}$ is the $L_{2}$-norm of a field weighted by the matrix $H^{-1}$. This interpretation of the finite element method leads to a natural way of determining the nodal stresses of a smoothed stress field: the vector $s$ can be obtained by substituting $\sigma$ with $\sigma$ in (7):

$$
\text { Minimize }\left|\sigma-\sigma_{h}\right|_{H}^{2}
$$

This is called the inverse finite element method, which can be rewritten in a stress projection form with $\boldsymbol{P}=\boldsymbol{H}^{-1} \boldsymbol{M}$ :

$$
\int_{\Omega}\left(\sigma-\sigma_{h}\right)^{r} H^{-1} M d \Omega=0
$$

The above projection form can further be transformed into a linear system of equations of dimension $m_{\text {nod }} \cdot n_{\mathrm{o}}$, where $n_{\mathrm{o}}$ denotes the total number of stress components:

$$
\boldsymbol{F} \boldsymbol{s}=\boldsymbol{d}
$$

where

$$
F=\int_{\Omega} M^{T} H^{-1} M d \Omega \quad ; \quad d=\int_{\Omega} M^{r} H^{-1} \sigma_{h} d \Omega
$$

This procedure of estimating the nodal stresses is in fact a generalized global least squares method where the inverse stress-strain matrix is taken as a weighting matrix. When this matrix is diagonal, the system (10) is reduced to $n_{\sigma}$ sub-systems, each of them containing only $m_{\text {nod }}$ equations. Here the inverse finite element method is identical with the global least squares method presented in the following paragraph. .

\subsubsection{Global $L_{2}$-projection}

Similarly to (8), another possible way of determining the vector $s$ is:

Or, in a stress projection form with $\dot{P}=\boldsymbol{M}$ :

$$
\text { Minimize } \mid \sigma-\sigma_{k} I_{2}^{2}=\int_{\Omega}\left(\sigma-\sigma_{k}\right)^{T}\left(\sigma-\sigma_{k}\right) d \Omega
$$

$$
\int_{\Omega}\left(\sigma-\sigma_{h}\right)^{T} M d \Omega=0
$$

This is called the global $L_{2}$-projection method of stresses, which leads to $n_{\sigma}$ linear systems of equations of dimension $m_{\text {nod }}$, each for one stress component:

$$
\boldsymbol{A} s=\boldsymbol{b}
$$

This method, first proposed in [4] under the name of conjugate stress method, seems the first stress smoothing method different from the methods of simple averaging. The smoothed stress field was initially called consistent stress field, since the matrix $A$ is similar to a consistent mass matrix used in the dynamical analysis of structures.

The global least squares method is cheaper but its accuracy is not lower compared to the inverse finite element method, since generally the stress-strain matrix is diagonally dominant. However, a global stress smoothing method is not suitable for post-processing. This is why Oden and Reddy have later proposed to use it only for regions of high stress gradients [5]. 
Hinton and Campbell have independently implemented the above method but they have systematically chosen the stress smoothing functions of degree one time lower than that of the displacement shape functions [6]. Such choice is not convenient for elements of degree one.

\subsubsection{Lumped-mass method}

One way to improve the efficiency of the global least squares method is to use the lumped form of the marix $A$ of (14), as in dynamic analysis. The lumped form of $A$, denoted by $\bar{A}$, is diagonal so that the determination of the nodal stresses is reduced to simple divisions. This is called the lumped-mass method. For 6-node triangles, special luniping procedures should be used to avoid zeros in the diagonal of the matrix $\bar{A}$.

Note that, for a mesh composed of linear elements, the lumped-mass method is identical with a method of nodal averaging of stresses weighted by element volumes. Moreover, if the mesh is uniform, the lumped-mass method is identical with the method of simple nodal (elemental) averaging. Numerical experiments have shown that the lumped-mass method cannot be more reliable than the methods of simple averaging.

\subsubsection{Local $\mathrm{L}_{2}$-projection}

Hinton and Campbell $[6,7]$ have also proposed an $L_{2}$-projection method but at an element level:

$$
\text { Minimize }\left|\sigma-\sigma_{k}\right|_{L_{2}(\Omega)}^{2}, \quad i=1, \ldots, n_{\text {els }}
$$

This leads to a continuous stress field inside each element, characterized by the set of nodal stresses $s\left(\Omega_{i}\right)$. Then, the simple nodal averaging procedure is used to obtain a smoothed stress field at the global level. Generally the smoothed stress field is of degree one time inferior to that of the displacement shape functions.

For 8-node parallelograms, the minimization procedure (15) is identical with a procedure of linear extrapolation of stresses sampled at the $2 \times 2$ Gauss points, which are also the superconvergent points for the stresses. In this special case, the nodal stresses can be evaluated by the following formula

$$
\left(\begin{array}{l}
s_{1} \\
s_{2} \\
s_{3} \\
\left.s_{4}\right)_{h}
\end{array}\right]_{1}=\left(\begin{array}{cccc}
1+\sqrt{3} / 2 & -1 / 2 & 1-\sqrt{3} / 2 & -1 / 2 \\
-1 / 2 & 1+\sqrt{3} / 2 & -1 / 2 & 1-\sqrt{3} / 2 \\
1-\sqrt{3} / 2 & -1 / 2 & 1+\sqrt{3} / 2 & -1 / 2 \\
-1 / 2 & 1-\sqrt{3} / 2 & -1 / 2 & 1+\sqrt{3} / 2
\end{array}\right)\left(\begin{array}{l}
\sigma_{k}^{\prime \prime} \\
\sigma_{k}^{\prime \prime} \\
\sigma_{k}^{\prime \prime} \\
\sigma_{k}^{\prime \prime}
\end{array}\right)_{h}
$$

where $\sigma_{h}^{\prime}(\Omega)(l=I, I I, I I I, I V)$ are the finite element stresses at Gauss point $l$. In the authors' opinion, this formula can be safely used for general 8 -node quadrilaterals.

The local $L_{2}$-projection method has been found to be more reliable than the global one especially for elements of degree $2[8]$.

\subsubsection{Loubignac-Cantin-Touzot iterative procedure}

Loubignac, Cantin and Touzot have proposed a quite different method of constructing a continuous stress field by an iterative procedure where the smoothed stress field tends to satisfy the nodal equilibrium conditions. The procedure is summarized as follows [9]:

1. solve the problem by the classical method to obtain the nodal displacement vector $q^{(0)}$ and the direct finite element stress field $\sigma_{k}^{(0)}$ as indicated by (3) and (4): 


$$
q^{(0)}=K^{-1} g \quad ; \quad \sigma_{k}^{(0)}=T q^{(0)}
$$

2. define nodal stresses by the method of simple nodal averaging as in $\$ 1.3 .2$;

3. interpolate the stresses from these nodal values to obtain a continuous stress field $\partial$;

4. evaluate the nodal equilibrium residuals of $\approx$ :

$$
\Delta \boldsymbol{g}=\boldsymbol{g}-\int_{\Omega} d i v \sigma d \Omega
$$

5. solve numerically the residual equations:

$$
\Delta q=K^{-1} \Delta g
$$

6. update $q$ and $\sigma_{h}$ :

$$
q^{(1)}=q^{(0)}+\Delta q \quad ; \quad \sigma_{1}^{(1)}=T q^{(1)}
$$

7. go to step 2 if convergence is not observed.

Normally, $K^{-1}$ and $g$ are available as finite element results. So, the solution of the residual equations is straightforward and cost effective. In the authors' opinion, the convergence is generally fast and 2 to 6 iterations are normally used. It is possible to speed up the convergence by using imposed surface traction on the corresponding boundaries.

It seems that the convergence depends strongly on the averaging procedure. Another difficulty in the procedure is the choice of a suitable stopping criterion.

The same authors later showed that this iterative procedure is equivalent to the solution of the same physical problem by using the classical finite element method with a modified set of shape functions [10]. It derives from a parent formulation that can be solved without iteration. The parent formulation contains the sum of two stiffness matrices. One is produced by the customary finite element shape functions. The other is produced from finite difference expressions for strain gradients that are not contained in the shape functions [11]. Because the parent formulation leads to a stiffness matrix whose bandwidth is larger than the classical one and the connectivity table relating elements and nodes is more complicated, it is more cfficient to use the iterative procedure.

\subsubsection{Superconvergent patch recovery}

Numerical experiments with finite element method show that for some classes of problems the displacements are more accurate, sometimes even exact, at the nodes of the mesh. This property is often designated as the nodal superconvergence of displacements [12].

Based on the property of nodal superconvergence of displacements and by using a method of Taylor series expansion, superconvergent points of stresses can be found inside, or at the boundary of, each element.

Zienkiewicz and Zhu have proposed a stress smoothing method based on a superconvergent patch recovery technique [8]. In this technique, stresses are evaluated at the superconvergent points of each element. Then, for each nodal point, the approximate stress values $s_{j}$ are determined by a local least squares fitting from stresses at a set of nearest superconvergent points of the surrounding elements. This set of points is called stress recovery patch of the node. The construction for such patches for boundary nodes requires particular treatments. It seems that this method is not yet applicable for meshes composed of different types of elements, because they have different stress recovery patterns. It has been improved in [13] by adding equilibrium conditions.

\subsubsection{Averaging + extrapolation}

By introducing the nodal superconvergence assumption of the finite element displacement field, it is possible to find in each element some interior or boundary points, where the directly evaluated stresses can be used to estimate the stresses at some boundary points of the element by a procedure of weighted averaging, the weights being only related to the distances between these points. The points where the stresses are sampled are called optimal stress extrapolation points. For structural boundary nodes, some special extrapolation schemes are used to obtain better 
estimated nodal stresses.

This method, called "averaging + extrapolation", has been initially used for one and two dimensional elements [14]. It can be easily extended to three dimensional [15] and plate bending elements [16].

\subsection{Closure}

Most of the stress smoothing methods are listed in Table I, with their main characteristics. Of course, the list is not exhaustive but intends to represent all the possible methods. The following items are presented in the table:
authors:
who probably have first proposed or implemented the method;
degree: degree of the stress smoothing functions;
level:
level of the stress smoothing procedure;
theory:
the main base of the smoothing procedure.

Table I: Typical stress smoothing methods

\begin{tabular}{|l|l|l|l|l|}
\hline \multicolumn{1}{|c|}{ method } & \multicolumn{1}{|c|}{ authors } & \multicolumn{1}{|c|}{ degree } & \multicolumn{1}{|c|}{ level } & \multicolumn{1}{|c|}{ theory } \\
\hline inverse F.E.M. & Ainsworth, Craig & any & global & projection \\
\hline global $L_{2}$-projection & Oden, Brauchli, Reddy & $\mathrm{p}$ & global & projection \\
\hline global $L_{2}$-projection & Hinton, Campbell & $\mathrm{p}-1$ & global & projection \\
\hline local $L_{2}$-projection & Hinton, Campbell & $\mathrm{p}-1$ & local & projection \\
\hline lumped-mass method & Zienkiewicz, Zhu & $\mathrm{p}$ & local & projection \\
\hline iterative procedure & Loubignac, Cantin, Touzot & $\mathrm{p}$ & global & nodal equilibrium \\
\hline patch recovery & Zienkiewicz, Zhu & $\mathrm{p}$ & local & superconvergence \\
\hline averaging + extrapolation & Beckers, Zhong & $\mathrm{p}$ & local & superconvergence \\
\hline
\end{tabular}

\section{ERROR ESTIMATION BASED ON SMOOTHED STRESS FIELDS}

\subsection{Exact error and precision}

The exact discretization error can be measured in many norms, such as energy norm, $L_{2}$-norm of stresses, etc. For 2D problems these norms are equivalent, and the energy norm:

$$
\left|e_{h}\right|_{E}=\left|\sigma-\sigma_{h}\right|_{H}=\left[\int_{\Omega}\left(\sigma-\sigma_{h}\right)^{T} H^{-1}\left(\sigma-\sigma_{h}\right)\right]^{1 / 2}
$$

is the most meaningful one. For this reason the energy norm will be used as a standard error measure. The above absolute error can be used to define a global precision measured in the energy norm:

$$
\eta=\frac{\left|\sigma-\sigma_{h}\right|_{H}}{|\sigma|_{H}}=\frac{\left|e_{h}\right|_{E}}{|u|_{E}}
$$

which is more suitable for practical use than the absolute error. These definitions also can be used at the element level. 


\subsection{Estimated error and precision}

According to (21), a smoothed stress field $\approx$, when it is sufficiently reliable, can provide a natural way to estimate the exact error: by substituting the exact stress field $\sigma$ with the smoothed one, an estimated global error, denoted by $\varepsilon$, is obtained:

$$
\varepsilon=\mid \boldsymbol{\sigma}-\sigma_{h} \mathbf{I}_{H} \equiv\left[\int_{\Omega}\left(\boldsymbol{\sigma}-\sigma_{h}\right)^{T} H^{-1}\left(\delta-\sigma_{k}\right)\right]^{1 / 2}
$$

The corresponding estimated global precision is then:

$$
\eta=\frac{\left|\tilde{\sigma}-\sigma_{h}\right|_{H}}{\sqrt{\left|\sigma_{h}\right|_{H}^{2}+\left|\sigma-\sigma_{h}\right|_{H}^{2}}} \equiv \frac{\varepsilon}{\sqrt{\left|\sigma_{h}\right|_{H}^{2}+\varepsilon^{2}}}
$$

These definitions also can be used at the element level and the estimated error is evaluated by numerical integration.

A structure that contains different materials can be subdivided into sub-structures and the stress smoothing procedure is performed for each of them.

\subsection{Quality measures of an error estimator}

To check the quality of an error estimator, several criteria can be used. First, the estimated error should tend to zero as the mesh size reduces to zero, this is the consistency criterion:

$$
\lim _{n \rightarrow 0} \varepsilon=0
$$
Secondly, it is to be hoped that the ratio between the estimated and the exact errors, denoted by $\theta$, and often called
"effectivity index"

$$
\theta=\frac{\varepsilon}{\left|e_{h}\right|_{E}}
$$

be bounded, this is the equivalence criterion:

$$
0<\theta_{\min } \leq \theta \leq \theta_{\max }<\infty, \quad \forall h
$$

A still stronger requirement is that the estimated error be asymptotically exact or convergent

$$
\lim _{h \rightarrow 0} \theta=1
$$

This is called the criterion of asymptotical exactness. If $\theta_{\min }$ and $\theta_{\max }$ are both close to unity, the error estimator is said to be globally reliable. If $\theta_{\min }>1$ the error estimator is conservative. If $\theta_{\max }<1$ it is an optimist one.

A globally reliable error estimator may not necessarily be reliable at the element level. To measure the local reliability of an error estimator, a "uniformity index" can be introduced. It is denoted by $S D$, defined as the standard deviation of the elemental effectivity indices:

$$
S D \stackrel{\text { def }}{=}\left[\frac{1}{n_{\text {ele }}}{ }_{i=1}^{n} \overrightarrow{\bar{\theta}}\left(\bar{\theta}-\theta_{i}\right)^{2}\right]^{n}
$$

where $\bar{\theta}$ denotes the mean value of the elemental effectivity indices. A small value of $S D$ means that the error estimator is locally reliable.

Today, few error estimators can satisfy all these criteria, especially the criterion of asymptotical exactness. But, often the violation of this last criterion does not necessarily lead to unacceptable results, because numerical solutions are always obtained with a certain level of accuracy. The error estimators are then required to be sufficiently reliable only for a given range of precision. For the range of $1 \% \leq \eta \leq 10 \%$ and for conventional meshes, if $0.8 \leq \theta \leq 1.2$ and $0 \leq S D \leq 0.2$, the error estimator can be said to be sufficiently reliable. 
A sufficiently reliable error estimator is a key factor in an adaptive process when the purpose of the analysis is to obtain a prescribed global precision. The adaptive process stops when the estimated error level is smaller than the user prescribed one, denoted by $\bar{\eta}$ :

$$
\tilde{\eta} \leq \bar{\eta}
$$

However, the exact precision may be far from the estimated one, since:

$$
\eta=\frac{1}{\theta} \bar{\eta}
$$

It follows that, for a conservative estimator, the prescribed precision is satisfied but with excessive refinement. On the contrary, an optimist error estimator may lead to a solution not satisfying the prescribed precision.

In the past, many people suggested to use the finite element solution of degree $p+1$, denoted by $u_{k, p+1}$, to estimate the error of the solution of degree $p$, i.e.,

$$
e_{k} \approx u_{k, p+1}-u_{k}
$$

This leads to the following effectivity index

$$
\theta=\frac{\left|u_{h, \rho+1}-u_{h}\right|_{E}}{\left|u-u_{h}\right|_{E}}
$$

Although this method is not practical, it can be shown that the error estimator does satisfy the equivalence criterion (27) and, when the exact solution is sufficiently smooth, satisfies also the criterion of asymptotical exactness (28).

Indeed, the error convergence law for a uniform refinement of the meshes is [1]:

$$
\left|e_{k}\right|_{E} \leq C_{p} h^{\min a_{, p}}
$$

where $\lambda>0$ is the stress singularity order of the exact solution, $C_{p}$ is a constant independent of the mesh size, but depending on the degree of the elements and on the exact solution. When the mesh size is sufficiently small. the inequality sign in (34) can be replaced by an equal sign.

Then, for $p+1$, (34) becomes

$$
\mid u-u_{k, p+1} \|_{E} \approx C_{p+1} h^{\min (k, p+1)}
$$

where $C_{p}>C_{p+1}$. This leads to the following bounds by the triangle inequalities of a norm:

$$
\left|u-u_{h}\right|_{E}-\left|u-u_{h, p+1}\right|_{E} \leq\left|\boldsymbol{u}_{k, p+1}-u_{h}\right|_{E} \leq\left|u-u_{k}\right|_{E}+\left|u-u_{h, p+1}\right|_{E}
$$

First case: the solution is quite singular. This corresponds to $\lambda \leq p$, then

$$
0<1-\frac{C_{p+1}}{C_{p}}<\lim _{h \rightarrow 0} \theta<1+\frac{C_{p+1}}{C_{p}}<2
$$

so that the error estimator is bounded. Second case: the solution is relatively smooth. This corresponds to $\lambda>p$ then

$$
\lim _{n \rightarrow 0} \theta=1
$$

so that the error estimator is asymptotically exact.

In a similar way, using the finite element solution of a more refined mesh, denoted by $u_{k n}$, to estimate the exact error produces the following bounds to the effectivity index:

$$
0<1-\left(\frac{1}{2}\right)^{\min (\lambda, p)}<\lim _{h \rightarrow 0} \theta<1+\left(\frac{1}{2}\right)^{\min (\lambda, p)}<2
$$

Thus, the error estimator using the solution $u_{M Z}$ is equivalent to the exact error, and is more reliable for smoother exact solutions and for elements of higher degree. 


\subsection{Survey of error estimators based on smoothed stress fields}

Due to its simplicity of implementation and interpretation, error estimation based on smoothed stress fields has been largely used in engineering analysis. Many methods of stress smoothing have been numerically tested to show the reliabiliy of the derived error estimators (Table II). But, strict mathematical proof of the asymptotical exactness and the equivalence is established only for a few methods.

Table II: References on the numerical tests of the error estimators based on stress smoothing

\begin{tabular}{|c|c|c|c|c|c|c|c|c|c|}
\hline \multirow{2}{*}{ method } & \multirow{2}{*}{$\begin{array}{l}\text { typical } \\
\text { references }\end{array}$} & \multicolumn{3}{|c|}{ triangle: nodes } & \multicolumn{5}{|c|}{ quadrilateral: nodes } \\
\hline & & 3 & 6 & 10 & 4 & 8 & 9 & 12 & 16 \\
\hline \multirow{2}{*}{$\begin{array}{l}\text { simple nodal } \\
\text { averaging }\end{array}$} & [21] & & & & $\mathbf{Y}$ & & & & \\
\hline & [23] & $Y$ & $\mathbf{Y}$ & & $\mathbf{Y}$ & $\mathrm{Y}$ & & & \\
\hline \multirow{5}{*}{ global $L_{2}$-projection } & [17] & $\mathbf{Y}$ & $\mathbf{Y}$ & & $Y$ & & & & \\
\hline & {$[20]$} & $\mathrm{Y}$ & $\mathbf{Y}$ & & & & & & \\
\hline & [22] & $\mathbf{Y}$ & $\mathrm{Y}$ & $\mathbf{Y}$ & & & & & \\
\hline & [8] & $\mathbf{Y}$ & $\mathbf{Y}$ & & $\mathbf{Y}$ & $Y$ & $\mathbf{Y}$ & $\mathbf{Y}$ & \\
\hline & [18] & $\mathrm{Y}$ & $\mathbf{Y}$ & & $\mathbf{Y}$ & $\mathrm{Y}$ & & & \\
\hline \multirow{2}{*}{ local $L_{2}$-projection } & [20] & $\mathrm{Y}$ & $\mathrm{Y}$ & & & & & & \\
\hline & {$[8]$} & & & & & $\mathbf{Y}$ & $\mathrm{Y}$ & $Y$ & \\
\hline \multirow{2}{*}{$\begin{array}{l}\text { Loubignac-Cantin- } \\
\text { Touzot }\end{array}$} & [20] & $Y$ & $Y$ & & & & & & \\
\hline & [23] & $\mathbf{Y}$ & $Y$ & & $\mathrm{Y}$ & $Y$ & & & \\
\hline \multirow{2}{*}{ lumped-mass } & [20] & $\mathbf{Y}$ & $Y$ & & & & & & \\
\hline & {$[18]$} & $\mathbf{Y}$ & $\mathbf{Y}$ & & $\mathbf{Y}$ & $\mathbf{Y}$ & & & \\
\hline \multirow{2}{*}{$\begin{array}{l}\text { superconvergent patch } \\
\text { recovery }\end{array}$} & [8] & $\mathbf{Y}$ & $\mathbf{Y}$ & & $Y$ & $\mathbf{Y}$ & $\mathbf{Y}$ & $\mathrm{Y}$ & $\mathbf{Y}$ \\
\hline & [23] & $\mathbf{Y}$ & $\mathbf{Y}$ & & $\mathbf{Y}$ & $\mathbf{Y}$ & & & \\
\hline $\begin{array}{l}\text { averaging + } \\
\text { extrapolation }\end{array}$ & {$[18]$} & $\mathbf{Y}$ & $\mathbf{Y}$ & & $\mathbf{Y}$ & $\mathbf{Y}$ & & & \\
\hline
\end{tabular}

In [17], it has been demonstrated that, if the smoothed stress filed is obtained by the inverse finite element method (7) and satisfies in addition the boundary prescribed traction, then the global effectivity index of the derived error estimator is bounded:

$$
\sqrt{\frac{1}{1+C_{2}(u) h^{\theta}}-\frac{h^{*} \Lambda^{2} K^{-1}}{\left|\tilde{\sigma}-\sigma_{\alpha}\right|_{H}}} \leq \theta \leq \sqrt{\frac{1}{1-C_{1}(u) h^{a-\theta+1}}}
$$

where $C_{1}(u)$ and $C_{2}(u)$ are two constants depending only on the exact solution, $\delta, \alpha$ and $K$ are positive constants. $\Lambda$ is the $L_{2}$-norm of the equilibrium residuals derived from the smoothed stress field:

$$
\Lambda=|\operatorname{div} \delta+\bar{f}|_{h}
$$


where $\vec{f}$ is the prescribed body force field.

Expression (40) has the following consequences:

1. A necessary condition for the asymptotical exactness of the error estimator is $q \geq p$.

2. The effectivity index is always smaller than or equal to unity. So that the error estimator is an optimist one.

3. If the residual term $\Lambda^{2}$ is converging faster than the estimated error $\left|\delta-\sigma_{k}\right|_{H}^{2}$, the asymptotical convergence of the enor is guaranteed. This may be true when the exact solution is somewhat smooth and when uniform meshes composed of elements of odd degree are used. But, for elements of even degree, the residual term is generally dominant and it will not be expected that the estimated error be asymptotically exact.

4. It is not efficient to use the smoothing functions whose degree is much higher than that of the displacement shape functions. The choice of $q=p$ is the most efficient while maintaining the requirements for convergence.

The above theoretical result is easy to extend to the case of the error estimator where the smoothed stress field is obtained by the global $L_{2}$-projection method, which is different from the inverse finite element method only by a weighting matrix. By using an eigenvalue analysis, it can be shown that both methods are equivalent and the following bounds can be obtained for the error estimator based on the global $L_{2}$-projection method

$$
\sqrt{\frac{1}{1+C_{2}(u) h^{\delta}}-\frac{h^{\alpha} \Lambda^{2} K^{-1}}{\left|\delta-\sigma_{h}\right|_{H}}} \leq \theta \leq \sqrt{\frac{\lambda_{\operatorname{man}} \lambda_{\min }^{-1}}{1-C_{1}(u) h^{--p+1}}}
$$

where $\lambda_{\max }$ and $\lambda_{\min }$ are the greatest and smallest eigenvalues of the stress-strain matrix respectively. Relations (40) and (42) show that both error estimators should give very similar results of error estimation.

The lumped-mass method is a simplified version of the global $L_{2}$-method thus may not be asymptotically exact. The global and local $L_{2}$-projection methods of Hinton-Campbell correspond to $q=p-1$, thus they cannot be expected to be asymptotically exact.

The Loubignac-Cantin-Touzot procedure reduces the residual term of the smoothed stress field. For elements of odd degree, the residual term is generally negligible compared to the estimated error, so that the use of the LoubignacCantin-Touzot procedure can only give marginal improvements to the reliability of the error estimator. But, for elements of even degree, the residual term is generally dominant and the Loubignac-Cantin-Touzot procedure could improve the reliability of the error estimator.

Numerical examples show that the methods based on the superconvergence assumption lead to more reliable error estimates than those based on the stress projection. But, the asymptotical convergence is not always observed, especially for elements of degree higher than one.

\section{BEHAVIOUR OF THE AVERAGING + EXTRAPOLATION ESTIMATOR}

\subsection{Presentation of the examples}

To examine the reliability of the error estimator based on the smoothed stress field obtained by the procedure "averaging + extrapolation", 4 numerical examples with increasing order of stress singularity have been analyzed (Table III, Figures 1 and 2). The convergence of the reliability measures of the error estimator is shown in Figures 3 to 7 . The successive meshes are obtained from the initial ones by uniform refinement. 
Table III: Characteristics of the numerical examples

\begin{tabular}{|c|c|c|}
\hline Problem & Data & Exact solution \\
\hline $\begin{array}{l}\text { a) Rectangular beam } \\
\text { subjected to bending } \\
\text { and shearing forces }\end{array}$ & $\begin{array}{l}\mathrm{E}=3 \cdot 10^{7}, \mathrm{v}=0.3, \mathrm{t}=1 \\
\text { plane stress }\end{array}$ & $\begin{array}{l}\left\{\begin{array}{l}\sigma_{x}=46.875 x y \\
\sigma_{y}=0 \\
\tau_{x y}=46.875\left(2-0.5 y^{2}\right)\end{array}\right. \\
0.5|\sigma|_{n}^{2}=0.0398333\end{array}$ \\
\hline $\begin{array}{l}\text { b) Hollow infinite plate } \\
\text { subjected to } \\
\text { unidirectional uniform } \\
\text { pressure }\end{array}$ & $\begin{array}{l}E=1000, v=0.3 \\
\text { plane strain }\end{array}$ & $\begin{array}{l}\left\{\begin{array}{l}\sigma_{x}=1-\frac{1}{r^{2}}\left(\frac{3}{2} \cos 2 \theta+\cos 4 \theta\right)+\frac{3}{2 r^{4}} \cos 4 \theta \\
\sigma_{y}=-\frac{1}{r^{2}}\left(\frac{1}{2} \cos 2 \theta-\cos 4 \theta\right)-\frac{3}{2 r^{4}} \cos 4 \theta \\
\tau_{x y}=-\frac{1}{r^{2}}\left(\frac{1}{2} \sin 2 \theta+\sin 4 \theta\right)+\frac{3}{2 r^{2}} \sin 4 \theta\end{array}\right. \\
0.5|\sigma|_{\Omega}^{2}=0.00769365\end{array}$ \\
\hline c) L-shaped domain & $\begin{array}{l}E=1, v=0.3, t=1 \\
\text { plane stress }\end{array}$ & $0.5|\sigma|_{\Omega}^{2}=15566.460$ \\
\hline $\begin{array}{l}\text { d) Crack problem in } \\
\text { linear elasticity }\end{array}$ & $\begin{array}{l}E=1, v=0.3, t=1 \\
\text { plane stress }\end{array}$ & $0.5|\sigma|_{\Omega}^{2}=8085.7610$ \\
\hline
\end{tabular}

Note that problem a) [18] and problem b) $[1,8]$ have analytical solution, so that the effectivity indices and uniformity indices can be exactly evaluated. For problems c) [19, $20,8,18]$ and d) $[18]$ the exact strain energies are estimated by the dual analysis method and the Richardson's extrapolation by using highly refined uniform meshes. so that only the global effectivity indices can bc approximately evaluated.
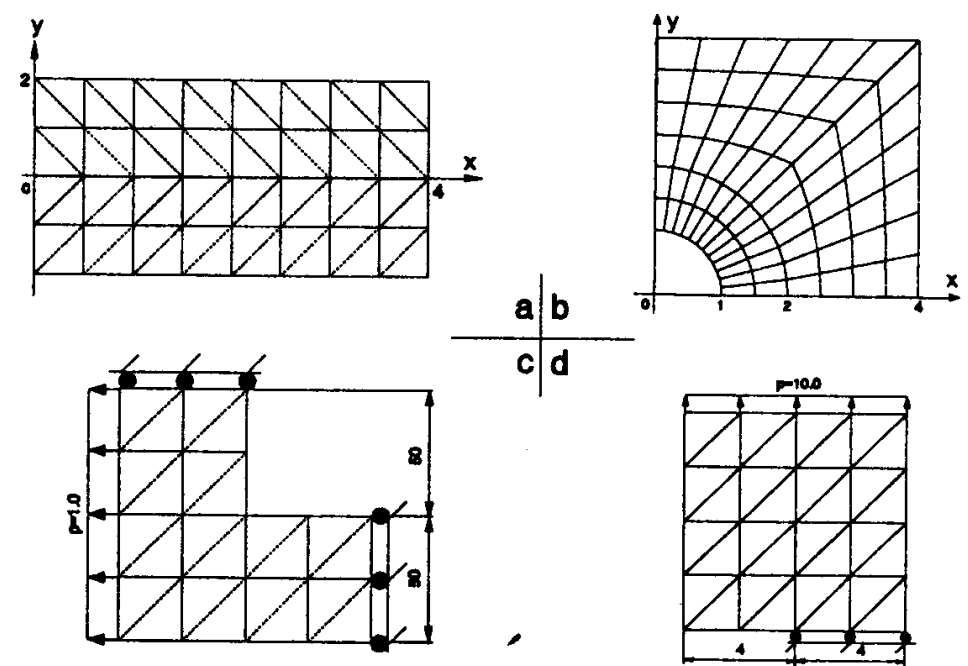

Figure 1: Uniform initial meshes for the 4 examples 


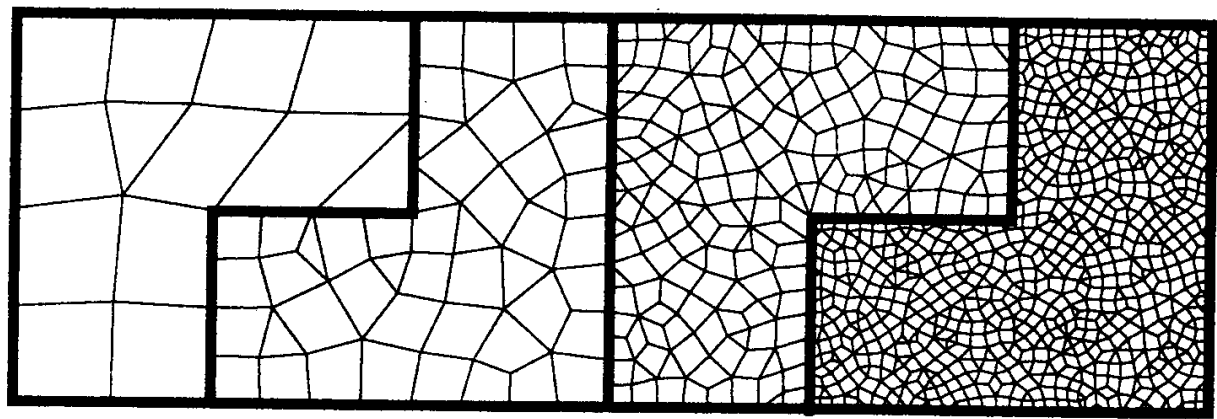

Figure 2: General meshes for the L-shaped domain

In the following figures, symbols $\square, \Delta$ and $\diamond$ represent pure quadrilateral, pure triangular and general meshes, respectively. Solid, long dashed and dotted lines represent elements of degree one, two and three, respectively.
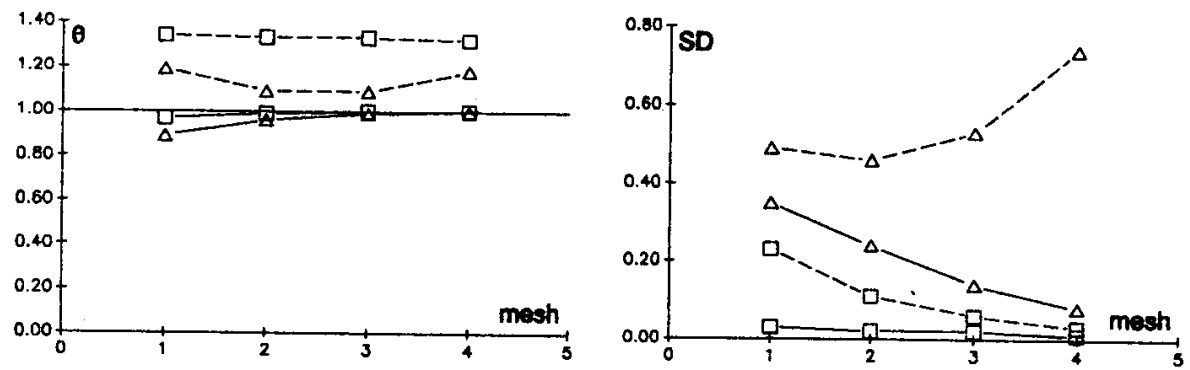

Figure 3 : Rectangular beam - convergence of the reliability indices
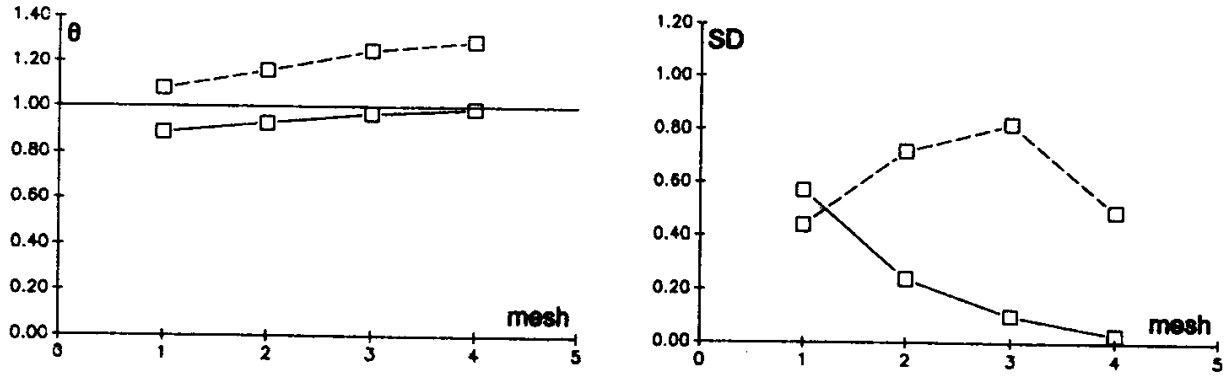

Figure 4 : Hollow infinite plate - convergence of the reliability indices

\subsection{Analysis of the results}

In these examples, the results show that the error estimator using a smoothed stress field obtained via the technique of averaging and extrapolation has the following characteristics:

1. It is less reliable for triangular and general meshes, as shown in Figures 3,5 and 6 . This is because here, the 
finite element solutions are less superconvergent. 2. For elements of degree 2 , it is less reliable for smooth exact solutions (Figure 3) than for more singular exact solutions (Figures 5 and 6). For a smooth exact solution, the finite element displacement field does not exactly interpolate the exact one. So, the stress field smoothed by the procedure "averaging and extrapolation" is only an approximation of the exact stress field. This leads to an over-estimation of the exact error for elements of degree 2. For a singular exact solution, the over-estimation due to the superconvergence assumption is balanced by the under-estimation due to the singularities, leading to a good estimation of the global error.

3. It can be unreliable for very coarse meshes. For a single element mesh, it is impossible to smooth the stress field. For a mesh with a single element layer, the stress extrapolation schemes cannot get sufficient information.

4. Its convergence is not guaranteed for any problem or mesh, especially for elements of degree higher than 1.

5. It is sufficiently reliable for meshes of usual levels of refinement. The global effectivity indices range generally from 0.6 to 1.4 , depending on elements type and degree.

6. It is applicable for general meshes composed of elements of degree up to 3. More specifically,

it can be applied to curved-edge elements. Note that some methods of error estimation can only be applied for pure triangular or pure quadrilateral meshes. Methods related to the equilibrium defaults of finite element solutions cannot generally treat curved-edges elements.

7. Its evaluation is very cheap. The boundary conditions of the physical problem are not required. The computation of the stresses at the optimal extrapolation points refers to a standard post-processing operation. The smoothing operation of stresses includes weighted averaging and polynomial extrapolation. The integration of the estimated element error is similar to that of the element stiffness matrix.

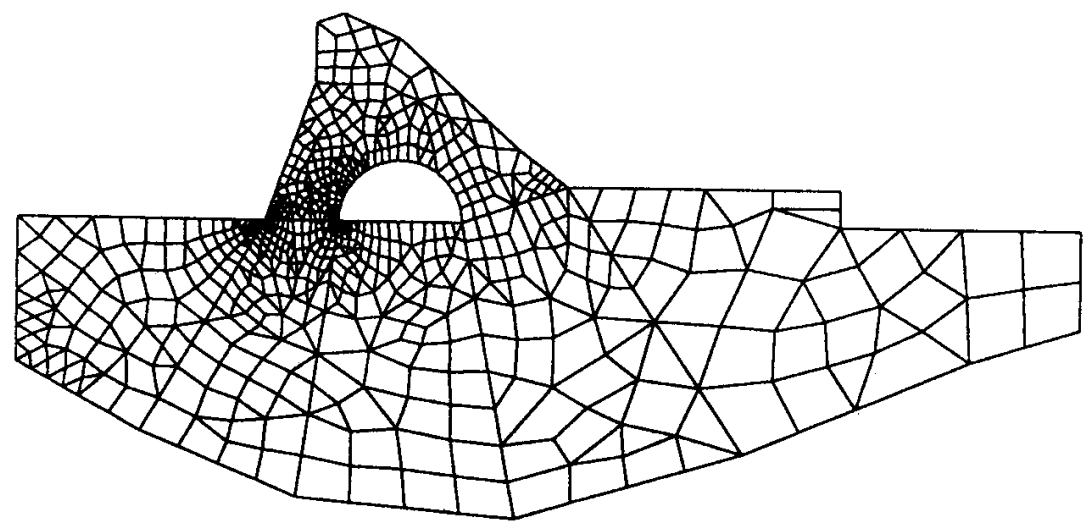

Figure 7 : Final mesh, degree 2, 5000 D.O.F. 


\section{ADAPTIVITY ILLUSTRATED BY THE TEST OF A HOLLOW DAM}

The structure shown in Figure 7 is modeled as a plane strain problem [19]. The goal of the analysis is to obtain a solution whose global prescribed precision in energy norm is about $3 \%$. Different mesh refinement strategies will be used and their efficiency compared.

Because the initial precision is already high $(8.8 \%$ ), and because elements of degree 2 are used, no intermediate mesh is needed. The final mesh, that ensures the prescribed precision, is shown in Figure 7. It contains many fewer elements and D.O.F. than does the one composed of elements of degree 1.

\section{precision ( \%)}

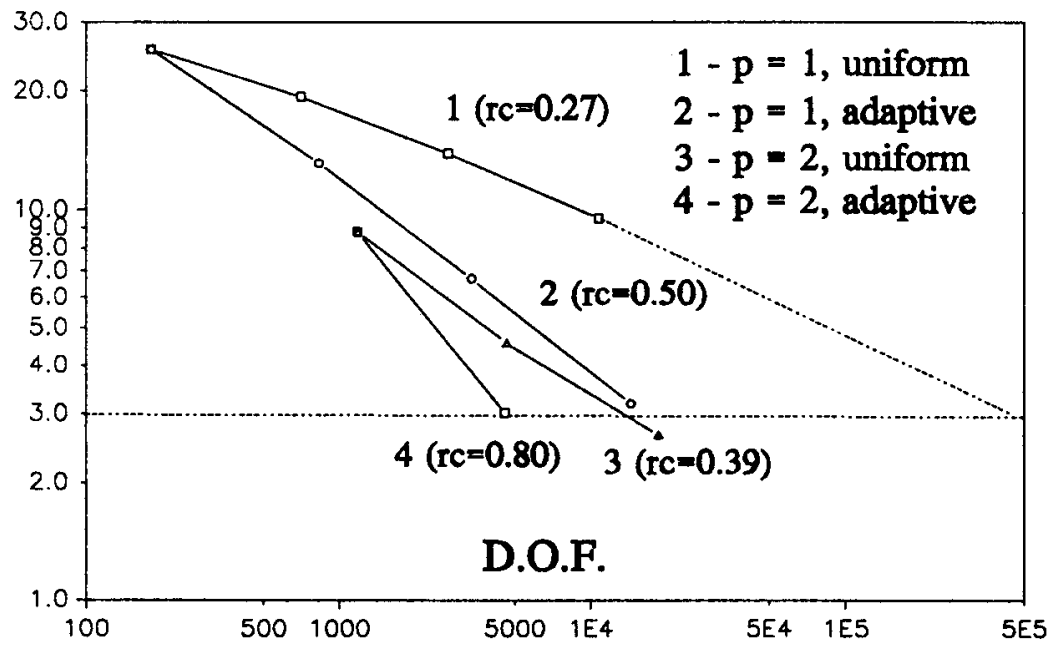

Figure 8 : Error convergence curves

The uniform mesh refinement strategy was also tested. All the convergence curves are shown in Figure 8. For uniform refinement, the asymptotic convergence rate is dominated by the most severe singular order of 0.273 . It also shows that the most economical way of obtaining a prescribed precision of $3 \%$ corresponds to the adaptive refinement strategy using elements of degree 2 . It requires only 2 analyses and a final mesh with only about 5,000 D.O.F. On the opposite, if uniform refinement strategy and elements of degree 1 are used, about 7 analyses are needed, and the final mesh would contain more than 500,000 D.O.F.

\section{CONCLUSIONS}

Using smoothed stress fields to estimate the discretization error of finite element solutions is one of the most widely used methods. It is the simplest method from both the implementation and evaluation points of view. But, the reliability of the derived error estimator depends on the quality of the recovered nodal stresses. For this purpose, methods based on the superconvergence assumption of finite element solutions are preferable to those based on the stress projection. The stress smoothing procedure "averaging + extrapolation" has been found to be quite suitable for error estimation. Moreover, it offers a continuous stress field that is generally of better quality than the finite element stresses. This continuous stress field can be directly used for visualization. 


\section{REFERENCES}

1. $\quad$ Szabó B.A. \& Babuska I., "Finite element analysis", John Wiley \& Sons, New York (1991).

2. Babuska I. \& Miller A., "The post-processing approach in the finite element method - Part I: Calculation of displacements, stresses and other higher order derivatives of the displacements", Int. J. Num. Meth. Engng., Vol.20, 1085-1109 (1984).

3. Stein E. \& Ahmad R., "On the stress computation in finite element models based upon displacement approximations", Comp. Meth. Appl. Mech. Engng., Vol.4, 81-96 (1974).

4. Oden J.T. \& Brauchli H.T., "On the calculation of consistent stress distributions in finite element calculations", Int. J. Num. Meth. Engng., Vol.3, 317-325 (1971).

5. Oden J.T. \& Reddy J.N., "Note on an approximate method for computing consistent conjugate stresses in elastic finite elements", Int. J. Num. Meth. Engng., Vol.6, 55-61 (1973).

6. Hinton E. \& Campbell J.S., "Local and global smoothing of discontinuous finite eiement functions using a least squares method", Int. J. Num. Meth. Engng., Vol.8, 461-480 (1974).

7. Hinton E., Scott F.C. \& Ricketts R.E., "Short communications: Local least squares stress smoothing for parabolic isoparametric elements", Int. J. Num. Meth. Engng., Vol.9, 235-238 (1975).

8. Zienkiewicz O.C. \& Zhu J.Z., "The superconvergent patch recovery and a posteriori error estimates. Part 1: The recovery technique; Part 2: Error estimates and adaptivity", Int. J. Num. Meth. Engng., Vol.33, 13311382 (1992).

9. Loubignac G., Cantin G. \& Touzot G., "Continuous stress fields in finite element analysis", A.I.A.A. J., Vol.15, NO.11, 1645-1647 (1977).

10. Cantin G., Loubignac G. \& Touzot G., "An iterative algorithm to build continuous stress and displacement solutions", Int. J. Num. Meth. Engng., Vol.12, 1493-1506 (1978).

11. Cook R.D., "Loubignac's iterative method in finite element elastostatics", Int. J. Num. Meth. Engng., Vol.18, 67-75 (1982).

12. Barlow J., "Optimal stress locations in finite element models", Int. J. Num. Meth. Engng., Vol.10, 243-251 (1976).

13. Wiberg N.E. \& Abdulwahab F., "Patch recovery based on superconvergent derivatives and equilibrium", Int. J. Num. Meth. Engng., Vol.36, 2703-2724 (1993).

14. Zhong H.G., "Estimateurs d'erreur a posteriori et adaptation de maillages dans la méthode des éléments finis", PhD. Thesis, University of Liege (1991).

15. Dufeu E., Scibetta M. \& Beckers P., "Estimateurs d'erreur et adaptation de maillages en 3D", $3^{\text {tme }}$ Congrès de Mécanique Theorique et Appliqué, Liege, May 30 - 31 (1994).

16. Bouđi E.M., Beckers P. \& Zhong H.G., "Estimateurs d'erreur pour des eléments de plaques basés sur la théorie discrétisée de Kirchhoff", Actes de Conférence StruCoMe 92, Paris, 599-611 (1992).

17. Ainsworth M., Zhu J.Z., Craig A.W. \& Zienkiewicz O.C., "Analysis of the Zienkiewicz-Zhu a-posteriori error estimator in the finite element method", Int. J. Num. Meth. Engng., Vol.28, 2161-2174 (1989).

18. Beckers P., Zhong H.G. \& Maunder E.A.W., "Numerical comparison of several a posteriori error estimators for 2D stress analysis", Euro. J. Finite Elements, Vol.2, No.2, 155-178 (1993).

19. Zienkiewicz O.C. \& Zhu J.Z., "A simple error estimator and adaptive procedure for practical engineering analysis", Int. J. Num. Meth. Engng., Vol.24, 337-357 ( 1987).

20. Shephard M.S., Niu Q. \& Baehmann P.L., "Some results using stress projectors for error indication and estimation", in "Adaptive methods for partial differential equations", eds. Flaherty J.E., Paslow P.J., Shephard M.S. \& Vasilakis J.D., SIAM, Philadelphia, 83-99 (1989).

21 Robinson J., Maunder E.A.W. \& Ramsay A.C.A., "Some studies of simple error estimators", Part I, II, III Finite element news, issues 4, 5, 6 (1992).

22. Strouboulis T. \& Haque K.A., "Recent experiences with error estimation and adaptivity. Part I : Review of error estimators for scalar elliptic problems", Comp. Meth. Appl. Meth. Engng., Vol.97, 399-436 (1992).

23. Strouboulis T. \& Haque K.A., "Recent experiences with error estimation and adaptivity. Part II : Error estimation for h-adaptive approximations on grids of triangles and quadrilaterals", Comp. Meth. Appl. Meth. Engng., Vol.100, 359-430 (1992).

24. Beckers P. \& Zhong H.G., "Mesh adaptation for 2D stress analysis", Second International Conference on Computational Structures Technology, Athens, Greece, Aug. 30 - Sept. 1 (1994). 